This PDF is a selection from an out-of-print volume from the National Bureau of Economic Research

Volume Title: The Rate and Direction of Inventive Activity: Economic and Social Factors

Volume Author/Editor: Universities-National Bureau Committee for Economic Research, Committee on Economic Growth of the Social Science Research Council

Volume Publisher: Princeton University Press

Volume ISBN: 0-87014-304-2

Volume URL: http://www.nber.org/books/univ62-1

Publication Date: 1962

Chapter Title: Strategy and Organization in a System Development Project Chapter Author: Thomas A. Marschak

Chapter URL: http://www.nber.org/chapters/c2140

Chapter pages in book: (p. 509 - 548) 


\title{
Strategy and Organization in a System Development Project
}

\author{
THOMAS A. MARSCHAK \\ THE UNIVERSITY OF CALIFORNIA, BERKELEY
}

OF THE $\$ 3.7$ billion spent by private industry on research and development in 1953, about 4 per cent, the National Science Foundation estimates, ${ }^{1}$ went to basic research. ${ }^{2} \mathrm{~A}$ breakdown of the remainder by categories related to the degree of "appliedness" does not yet exist. It is safe to say, however, that a large proportion went to the development of what have come to be called systems.

There is no accepted unambiguous definition of a system. To call it a collection of interrelated components seems inadequate, for that is true of the simplest gadgets, down to the safety pin. Nor is it satisfactory to say that, compared to basic research, the uncertainties in developing a system are negligible and the goals precise. For, while a system is based on established principles which are the outputs of basic research, the difficulty of applying them to achieve a given performance goal may be highly uncertain, so that an initially selected goal may be substantially revised as development proceeds. Moreover, a system development project is generally larger than the average basic research project and more, in a sense, is at stake: in a system project a commitment may be based on a conjecture (about nature) whose uncertainty is negligible by the standard of basic research. But where, in a basic research project, a commitment might be an order for a dozen Bunsen burners, in a system project it is likely to be the task assignment of a large team of engineers, and sometimes it is heavy investment in production facilities. The uncertainties of system development may be niggling and prosaic compared to the deep mysteries of basic research, but it is urgent, all the same, to assess them carefully.

For our purpose it will suffice to think of a system as a complex aggregation of components, based on established principles. An aircraft is a system, and so are a color television set, a telephone exchange,

\footnotetext{
${ }^{1}$ Science and Engineering in American Industry, Final Report on a 1953-1954 Survey, National Science Foundation, 1956, Table A-13.

${ }^{2}$ Defined by the National Science Foundation as "Projects which are not identified with specific product or process applications, but rather have the primary objective of adding to the over-all scientific knowledge of the firm" (ibid, p. 18).
} 
and a synthetic fiber plant. The established principles permit the confident assertion that (1) for some effort, or (2) for no effort, however large, the system can be made to attain a given performance. Knowledge of the effort needed in case (1) varies widely in accuracy from system to system and so, consequently, does the precision with which performance goals are specified when development of the system begins.

There is no need to go into the normative and positive questions about industrial research and development (and hence about technical change) for which a better understanding of system development is important. A major one: what is "efficient" system development and is it observed in American industry? The empirical study of system development could take at least three forms: an analysis, industry by industry, of relevant aggregates; a comparison of the conduct of system development as between industrial laboratories; or an examination of a number of system development projects in a variety of laboratories. Data for all three approaches are difficult to obtain. We are concerned here with the third approach : the intensive historical study of completed system development projects.

A sufficient number of such studies, over a sufficiently wide range of industries and project sizes, could help to provide first answers as to what sorts of development strategies and what types of project organization are likely to be associated with "successful" system development. By strategies we mean rules for making decisions as the project proceeds and knowledge is gained. The problem of measuring success is, of course, very troublesome. We shall be content to focus on certain observable and avoidable difficulties whose presence in the course of a project indicates "inefficiency" or less than maximum success.

While the accumulation of case studies would help to isolate the causes of these difficulties, some theoretical work on the nature of systems and on good organization and strategy for system projects would be in order as well. ${ }^{3}$ For even without the peculiar role of uncertainty, a system would generally present a scheduling problemthe efficient scheduling of tasks, whose complexity goes beyond the existing results on scheduling problems arising in production. ${ }^{4}$ With the introduction of uncertainty and of objective functions defined over a space of system performance parameters, the problem of good

${ }^{3}$ A first try at some formal theory is under way.

E.g., on assembly-line scheduling. 
strategy and organization becomes a completely new one, and a formal statement of it, at the least, seems essential.

We shall not attempt it here. The present paper merely illustrates the possibilities of case studies for the support of informally stated hypotheses about the types of strategy and organization which avoid or make likely specific types of difficulties. One case study is presented which is consistent with one such hypothesis.

The system we shall consider was developed at the Bell Telephone Laboratories. It is a microwave relay system for the transmission over long distances, via a chain of relay towers, of telephone conversations and television signals. Its development was complete and its manufacture had begun in 1958. The start of its development as a system can reasonably be dated 1952. The project built up to a peak of about ninety technical persons, and its man-power and other direct costs are roughly estimated at $\$ 15,000,000$. In the Bell Telephone Laboratories it was one of the largest and most complex (nonmilitary) systems undertaken.

The materials available for the study were official project documents, informal memoranda and conference minutes, and some interviews. The written coverage of events was often sketchy and the time available for interviews to fill gaps was quite limited. It seems fair to say that for the major events in the project's history records exist (and were examined); for the others, the records and interviews covered, very roughly, a random sample. While it would be pleasant for the investigator of system development to come across a project in which a total history was kept-in which every idea, message, and decision was recorded on a punched card-one suspects that such a project would have drawbacks for the laboratory which, in its view, would outweigh the investigator's joy. If informality and partial independence of technical personnel are important attributes of successful projects, then, it may be conjectured, successful projects are far removed from the extreme of the punched card and are not easy to study. At any rate, even with the interest and cordial cooperation with which the study was received, much of the following account remains the writer's personal impression.

The following section gives a simplified layman's description of the system studied (known as the TH system). The second section is an "anonymous" history, concentrating on the general strategy of development and avoiding any mention of who was involved. It makes lavish use of the passive voice ("It was decided that ..."), 
and might be the history of a one-man project. It is, of course, by no means complete, in the sense that very many events are omitted, but the strategy of development seems fairly portrayed. The fourth section contains some observations about the organization of the project (who did what). The last section is a recapitulation and a brief statement of a hypothesis about strategy and organization of system projects with which the case of TH seems consistent.

\section{A Description of the TH System}

The transmission of telephone conversations and television broadcasts over long (transcontinental) distances has been achieved in two ways - by coaxial cable and by microwave relay. The second method, the later of the two to be developed, dominates the former-for a given investment cost it provides far greater capacity (with comparable performance). A microwave relay network is composed of towers or repeater stations, a constant distance apart, each of which contains equipment that receives very high-frequency signals from the adjacent stations, equipment that amplifies the signals received, and equipment that transmits the amplified signals to the adjacent stations. At each end of a link in the microwave relay network originating telephone messages and TV broadcasts are transformed into signals of very high (microwave) frequency.

The first microwave relay system-the TD-2 system-was put into transcontinental operation by the American Telephone and Telegraph Company in 1951. Its microwave signals have frequencies around 4,000 megacycles per second $(\mathrm{mc} / \mathrm{sec})$. The TH system, with which we are concerned, operates at frequencies around $6,000 \mathrm{mc} / \mathrm{sec}$. Its purpose is to greatly augment the capacity of TD-2, and it uses much equipment of totally new design. Its capacity is approximately 11,000 two-way telephone conversations (one television broadcast can be substituted for each 1,860 conversations)-roughly three times the capacity of TD-2.

We proceed now to a simplified description of $\mathrm{TH}$-by a layman for laymen - which will serve as a reference base for the historical and organizational accounts that follow. Figure 1 provides a schematic portrayal of the system.

Consider the transmission of telephone conversations and TV broadcasts, via TH, from one location to another. At the first location we find a telephone central office in which groups of originating 
FIGURE 1

Highly Simplified Schematic Diagram of TH System, Tracing Progress of One Signal, Covering One Channel

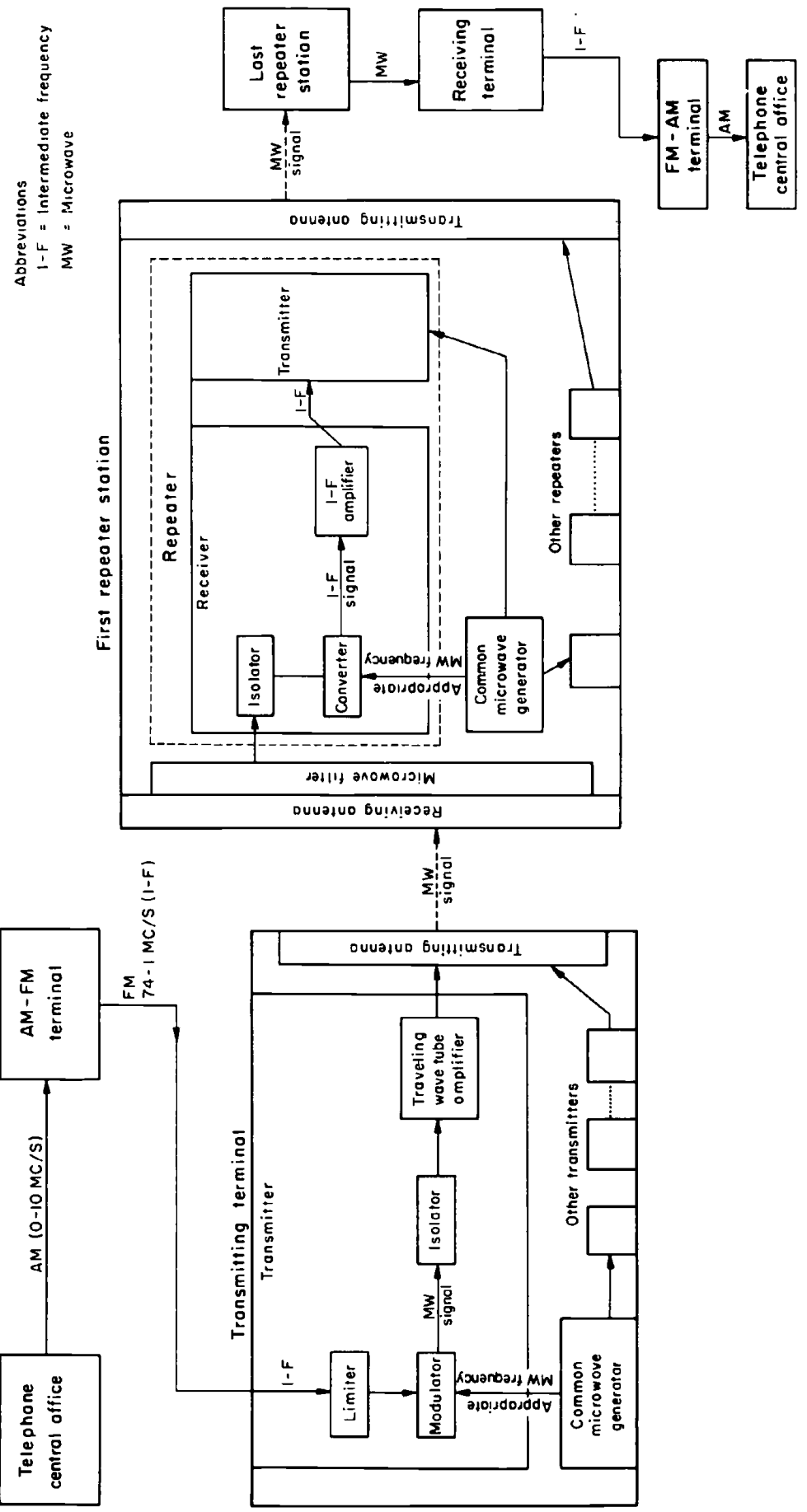


telephone conversations are transformed (by equipment developed some years before $\mathrm{TH}$ ) into amplitude-modulated radio signals. These signals, together with television signals (which are also AM), are sent via cable to an $A M-F M$ terminal. We shall trace the progress of one signal, conveying, say, a group of telephone messages (alternatively it could convey a TV program). The AM signal in question, like all the originating AM signals, uses the frequency band from 0 to $10 \mathrm{mc} / \mathrm{sec}$, called the base band. At the AM-FM terminal it is converted, by equipment developed for $\mathrm{TH}$, into a frequency-modulated signal whose center frequency is $74.1 \mathrm{mc} / \mathrm{sec}$, called the intermediate frequency of $i-f$. The band of frequencies about the i-f, containing the modulations of the $\mathrm{i}-\mathrm{f}$ which carry the group of messages in question, is 28 megacycles wide. This FM signal is fed, via cable, to a transmitting terminal.

Here the signal enters the transmitting modulator, where it is impressed on a microwave carrier signal, whose frequency is near 6,000 $\mathrm{mc} / \mathrm{sec}$; the microwave carrier signal, in other words, is frequency modulated so as to match (to carry the same information as) the incoming i-f FM signal. The source of the microwave carrier signal is a common microwave generator which provides all the microwave carrier frequencies needed in the transmitting terminal. The modulated microwave signal passes through a ferrite isolator (which attenuates undesired signals reflected back from subsequent components) and then goes to a traveling-wave-tube amplifier; the amplified signal finally reaches the transmitting terminal's transmitting antenna.

The microwave signal is detected at the first repeater station, containing a receiving antenna, several repeaters, and a transmitting antenna, and located some thirty miles away. Each repeater consists of a receiver and a transmitter; the components of the latter are identical with those just described for the transmitting terminal. After passing through the repeater station's receiving antenna, through an attached wave guide, and through a microwave filter which separates it from adjacent signals, the microwave signal goes to one of the repeaters. The first repeater component which it enters is an isolator (whose general purpose is as described above). It next goes to a receiving modulator or converter. Here it is impressed on a microwave signal of different frequency (obtained from the repeater's common microwave generator) in such a way that an FM signal at i-f emerges. The i-f signal contains the same information as the original received microwave signal, but like the received signal it is very weak. It is 
amplified in the $i-f$ amplifier. The amplified i-f signal enters a transmitter; the transmitter's components have already been described. ${ }^{5}$ The power needs of each repeater station are met by a power supply.

Following the final repeater station there is a receiving terminal, containing receivers (whose components have just been described). The amplified i-f signal which emerges is sent to an FM-AM terminal, where it is converted to the base band AM signal, and then sent to a telephone central office (or to a local TV transmitter if it conveys television).

The term "signal" needs to be made more precise. TH uses the 500-megacycle-wide microwave band between 5,925 and 6,245 $\mathrm{mc} / \mathrm{sec}$. This band is divided into 16 broad-band channels, each $28 \mathrm{mc}$ wide, or 8 two-way broad-band channels, each composed of 2 nonadjacent broad-band channels. Of the 8,6 are for regular service and 2 are protection channels. Each two-way broad-band channel will handle 1,860 two-way telephone conversations or one television program. ${ }^{6}$

It will be important for our purposes to clarify further the placing of the two-way channels within the $500 \mathrm{mc}$ band as well as their use. For the scheme of channel placement or frequency allocation was one of the major choices facing the developers of TH. Consider, for example, the two-way channel whose component channels have the carrier frequencies $6,226.9 \mathrm{mc} / \mathrm{sec}$, and $5,974.9$ $\mathrm{mc} / \mathrm{sec}$ when no messages are being transmitted. Now suppose the channel's complement of 1,860 telephone messages is sent from location A to location B via this two-way channel. This means that at the transmitting terminal a carrier signal of $5,974.9 \mathrm{mc} / \mathrm{sec}$ is frequency modulated-its frequency is altered at any instant of time above or below the carrier frequency so as to convey the information in these telephone messages. At the first repeater station, the frequencies conveying the messages are shifted upward (in the transmitting modulator) by $252 \mathrm{mc} / \mathrm{sec}$, so that the unmodulated carrier frequency becomes $6,226.9 \mathrm{mc} / \mathrm{sec}$ and the telephone messages now cause deviations above and below

SThe purpose of bringing the signal down to i-f at the repeater is to facilitate channel switching. See section below entitled "Generating Alternative Major System Characteristics Consistent with the Objectives."

- The width of each channel is determined by the width of the base band and (roughly) by the noise level to be tolerated in transmitting the information the base band signal contains. 
this frequency. At the second repeater station the frequencies conveying the messages are shifted down to the original band centered at $5,974.9 \mathrm{mc} / \mathrm{sec}$.

The alternation between the two components channels from repeater station to repeater station continues until the receiving terminal at B is reached. There is an analogous alternation in the opposite direction for the other part of the telephone conversation in question: the transmission of a message from $B$ to $A$. The result is that at any repeater station one $28-\mathrm{mc}$ frequency band carrying the telephone conversations in question is received from both directions and another (not adjacent to the first) is transmitted in both directions. For each two-way channel, there is, at a given repeater station, a distinct pair of transmitters and a distinct pair of receivers. All the transmitting frequencies in the repeater are grouped together and all the receiving frequencies are grouped together, the two groups being separated by a guard band $52 \mathrm{mc}$ in width. The main purpose of this frequency-shift scheme is to avoid over-reach interference, which results when two successive repeaters transmit the same frequency band in the same direction. Figures 2 and 3 portray the scheme.

Very efficient use of the entire $500 \mathrm{mc}$ band is made possible by cross polarization (portrayed in Figure 3). Any two adjacent one-way broad-band channels in a repeater station's transmitting group and any two in its receiving group are oppositely polar-

FIGURE 2

Frequency Shifting: One Two-Way Broad-Band Channel, Composed of One-Way Channels $F_{1}$ and $F_{2}$

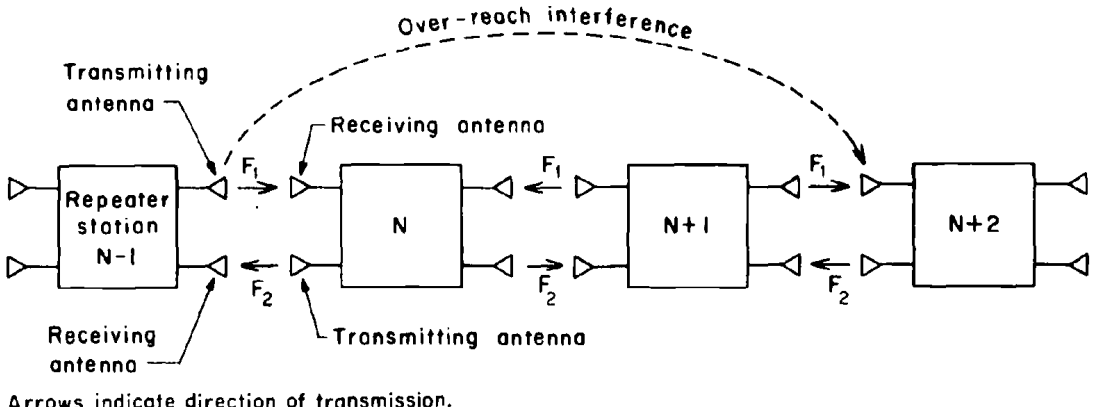

Arrows indicate direction of transmission.

Arrows indicate direction of transmission. 
FIGURE 3

Two-Frequency Plan of Frequency Allocation, Using Cross-Polarization

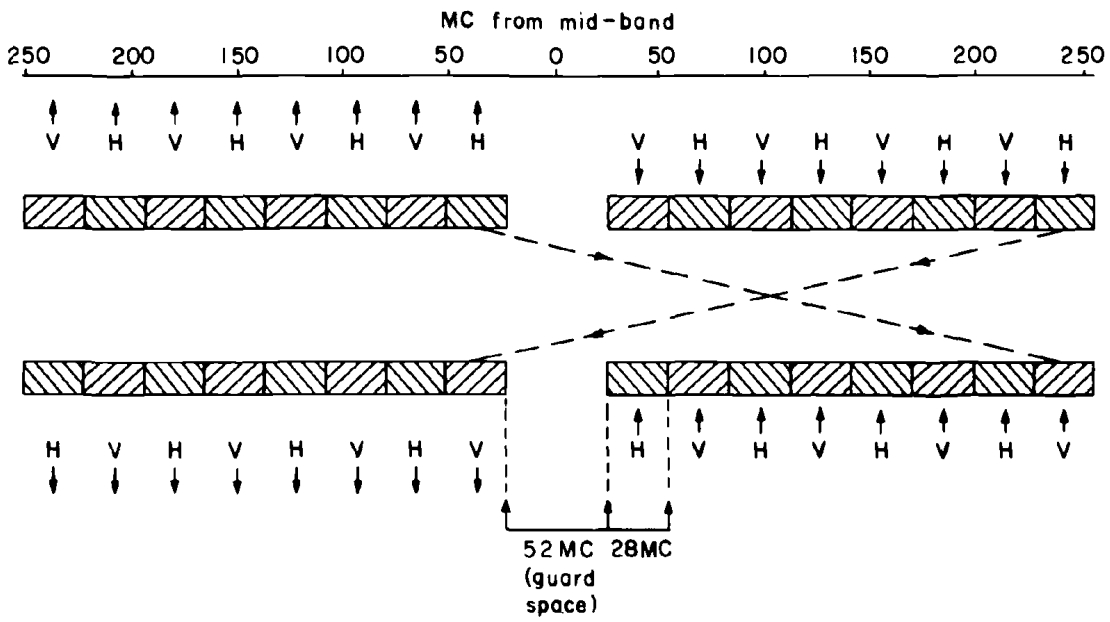

The crossing diagonal broken lines indicate a possible two-way brood-band channel of a given repeater station. Arrows indicate direction of transmission.

The crossing diagonal broken lines indicate a possible two-way broad-band channel at a given repeater station. Arrows indicate direction of transmission.

ized-if signals in the first channel travel in a horizontal plane to or from the next repeater, signals in the second travel in a vertical plane. This permits adjacent channels to be edge-to-edge without interference; without cross polarization there would have to be a guard band between two adjacent channels. Cross polarization also makes it possible to use one receiver and one transmitter for two adjacent (and oppositely polarized) oneway broad-band channels. Thus each repeater station contains in total eight repeaters (eight receivers and transmitters)-four for the eight one-way broad-band channels going in one direction and four for the eight going in the other direction.

\section{An Anonymous History of the TH Project}

\section{ORIGINS}

The TH system has, as we have seen, a predecessor- - the TD-2 microwave relay system, first put into transcontinental operation in 1951. TD-2 operates at frequencies between 3,700 and $4,200 \mathrm{mc} / \mathrm{sec}$. 
It has five two-way broad-band channels; each channel's capacity is now 600 telephone conversations or one TV broadcast. (Each channel's capacity is less, per mc of width, than in TH, primarily because the amplifier used has much less power than TH's traveling-wavetube amplifier.) The original transcontinental route has been paralleled and additional north-south and east-west fragments have been constructed so as to form a 25,000 mile network (densest in the eastern half of the country).

The growth in utilization of TD- 2 was predicted at its inception to be quite steep. After the first two years of operation it was predicted that the capacity of some of its major links would be exhausted by 1960 . The Federal Communications Commission had, in 1946, assigned to common carriers three microwave frequency bands for telephone and television transmission and to the Bell System the use of these three bands by transmitters at specific locations. One band was that used by TD-2. The remaining two were the 5,925-6,245 $\mathrm{mc}$ band and the $10,700-11,000$ mc band. For accepted technical reasons ${ }^{7}$ it was decided to use the latter band for a future short-haul feeder system and the former for the next long-haul relay system to be developed after TD-2.

It was clear at the time these decisions were made that a long-haul system operating in the $6,000 \mathrm{mc} / \mathrm{sec}$ range posed a major development task. Devices that accurately and reliably amplified signal channels in this range (each channel conveying a great deal of information) did not exist; there were at least two approaches to such devices and it was far from clear which was better. In addition to amplifiers, other important components of a future system were equally problematic. The future development task was conceived, moreover, as achieving an improvement over TD-2; it was to make more efficient use of its frequency band.

General exploratory work relevant to microwave transmission systems had been a continuing activity at Bell Laboratories for some years. Experimental tubes and other devices were constantly being built and investigated so as to provide an array of interesting component possibilities for unspecified future microwave systems. It seems to have been in 1949 , however, that work was first specifically directed toward the components and techniques which were then the major puzzles of a future $6,000 \mathrm{mc} / \mathrm{sec}$ long-haul system. The

\footnotetext{
'Namely, that the difficulties of using the very high frequency band would be less serious in short-haul service.
} 
years 1949-51 were primarily years of resolving fundamental uncertainties before the start of system planning and development.

\section{9-51: RESOLVING FUNDAMENTAL UNCERTAINTIES AND IDENTIFYING OBVIOUS BOTTLENECKS}

1. The most extensive uncertainties concerned traveling wave tubes for amplification in the transmitter of a microwave relay system. The principles of the traveling wave tube had been developed just after World War II (largely in Britain and at Bell Laboratories) and experimental models had been built as early as $1947 . .^{8}$ It seemed likely that traveling wave tube amplifiers with the required characteristics in the $6,000 \mathrm{mc} / \mathrm{sec}$ range could be developed. But it was highly uncertain how quickly the design of a satisfactory tube and an associated magnetic focusing structure could be found.

At the same time there existed an alternative to the traveling-wavetube amplifier-an amplifier based on a planar triode. Such an amplifier had been developed for TD-2. There was a possibility that the TD-2 triode could be modified ${ }^{9}$ to provide satisfactory amplification at $6,000 \mathrm{mc} / \mathrm{sec}$. Attempts to do so were still underway, on a small scale, in 1949. But toward the end of 1949 the consensus was that the traveling wave tube held the greater promise.

There was interest concurrently in a low-noise traveling wave tube, a tube specially designed to have a much superior noise figure but a substantially smaller gain and smaller power output than the tube intended for the main transmitting amplifier. The low-noise tube would be used in the receiver to amplify the received microwave carrier signal before its conversion to an i-f signal in the converter. This promised to decrease significantly each repeater's contribution to the total noise in any future system in which signals were brought

${ }^{8} \mathrm{~A}$ very crude description of the tube is as follows. An electron gun, a helix (a coil of wire), and a collector are sealed in.a long, thin glass tube. An electromagnetic wave to be amplified enters the tube through an input wave guide. The wave travels around the helix towards the collector with almost the speed of light; its effective speed through the tube, however, is less, roughly equalling the speed of the electrons which are sent through the helix from the gun by a voltage close to 2,000 volts. (The helix, in other words, "slows down" the electromagnetic wave by making it traverse a lengthy route to get from one end of the tube to the other.) The electrons and the wave travel along together, with the wave acting on the beam of electrons to group them in clusters which "match" the wave and which, in turn, act on it so as to amplify it. The waves can be visualized as waves on water and the electron stream as a breeze, which blows past the waves and raises them higher and higher as they travel. The amplified signal leaves the tube through an output waveguide. The direction of the electron stream must be very accurately maintained, and this is the function of a magnetic focussing structure surrounding the tube.

- Essentially by bringing the grid closer to the cathode. 
down to an i-f at repeaters. Both traveling wave tubes under study through late 1951 were $4,000 \mathrm{mc} / \mathrm{sec}$ tubes built to match the only test equipment then available. It was expected that information obtained from tests at these frequencies could later be applied to higher frequency tubes. Only at the end of 1951 did work begin on 6,000 $\mathrm{mc} / \mathrm{sec}$ versions of the two tubes.

2. Cross-polarization studies got under way in 1949. Cross polarization was known to have promise as a means for making more efficient use of a given microwave frequency band and experimental data were needed to estimate the difficulties of incorporating the techniques in the future system. Knowledge of waveguide and antenna designs permitting efficient cross polarization was obtained.

3 . Frequency allocation studies were begun, to consider alternative ways of dividing up a future system's frequency band among channels and to deduce some of the broad requirements that each alternative would impose on the system.

4. Effort in the years 1949-51 was also directed at identifying and starting work on obvious potential bottlenecks which would arise in developing a future system unless work on them preceded the start of system development. By obvious bottlenecks we mean those that can be prevented by performing (before the start of system development) straightforward tasks whose magnitude is predictable with high confidence. Such tasks require no innovations and are independent of those properties of the final system which are initially very uncertain.

The chief obvious potential bottlenecks were certain items of test equipment. Conferences in 1949 formally emphasized the importance of these items. The highest-frequency test equipment then existing was in the $4,000 \mathrm{mc} / \mathrm{sec}$ range (largely developed for TD-2). The absence of $6,000 \mathrm{mc} / \mathrm{sec}$ test equipment was already constraining experimental traveling wave tube work. It would literally halt important work once a future system was in full development. To produce a variety of test equipment at $6,000 \mathrm{mc} / \mathrm{sec}$, while a fairly straightforward engineering task, was apparently one that was sure to require substantial minimum time periods - up to a year and a half for some items. Allocation of more manpower to test-equipment development within the anticipated manpower limits could not, apparently, reduce the time required below these minima. Work on some of the major items consequently started in 1950 and 1951. 


\section{2: PRELIMINARY SYSTEM PLANNING}

In 1952 the planning of TH as a system began. The official authorization of preliminary planning meant that the accumulated information about the difficulty of developing $\mathrm{TH}$, gained in the previous three years' attack on the system's fundamental puzzles, showed it to be certainly worth another one or two years' work at a great but still relatively low level of intensity. ${ }^{10}$ At the end of the period it would be decided whether, in view of the predictions then possible about the roughly planned system and its development effort, and in view of the rising pressure on TD-2, a commitment to full-scale development should be made. A (partial) alternative still remaining at the time of that decision would be construction of additional TD-2 routes.

As a first step in planning, it was decided that TH would use the routes and towers of TD-2 and that the components of TD-2 should be used or adapted whenever possible. As a result of the work of 1949-51 it was also decided that microwave amplification would be based on the traveling wave tube; that cross polarization would be used together with an antenna type explored in 1949-51;11 and that shifting of frequencies would occur at repeaters, as had been the case in TD-2. ${ }^{2}$

There then remained, at this stage, three major planning problems: formulating broad objectives; identifying alternative sets of major system characteristics consistent with these objectives; and formulating broad requirements for certain "key" components (those likely to require a long development time, or those that must be nearly completed before the development of important components can start). We shall discuss these problems in order.

\section{Objectives}

The system's purpose is to transmit information; its success in doing so depends, essentially, on the number of messages that can be simultaneously transmitted over any link in the system, the total noise expected over any link, and the reliability of any link. For a given

\footnotetext{
${ }^{10}$ Roughly 78 technical man-years were spent in 1952-1954 out of an estimated total of 345 spent on TH development by 1958. Further data on time path of expenditure are given at the end of this historical section.

"Horn-reflector antennas had been shown during this period to be much more efficient, especially for cross-polarization, than the delay-lens antennas of TD-2.

${ }^{12}$ But an important choice remained as to how many frequency bands each channel used in each directior.. See below.
} 
development effort and a given production and operating cost of the developed equipment, there is, in general, some scope for trade-off among these three magnitudes. For example, if each telephone conversation were assigned a larger portion of a two-way broad-band channel, a better signal-to-noise ratio could be attained, but fewer conversations could then be fitted into the given $500 \mathrm{mc}$ band. To some extent, moreover, a greater development effort and a greater production and operating cost can "buy" better values of one or more of the three magnitudes.

In the early planning of the $\mathrm{TH}$ system, the objective with respect to noise was to meet standards previously set by the Bell System for long-haul telephone and television circuits. These specify a maximum acceptable total noise level for any long-haul link. ${ }^{13}$ There was a corresponding goal with respect to messages transmitted: a minimum of six two-way broad-band channels each carrying at least 1,800 (two-way) telephone conversations or one TV broadcast. ${ }^{14}$ The reliability goal was less precisely stated: a "very high" degree of reliability was to be obtained by making use of protection channels (two were planned) in addition to the six normal two-way broad-band channels. In the event of a breakdown in an item of equipment on which a given normal channel depends, one of the protection channels would be switched to automatically. ${ }^{15}$ Alarm and control circuits would be used to signal the difficulty to maintenance personnel.

How binding were these objectives? The noise objective seems to have been the most critical. Even here, however, the view was certainly not that the entire development would be a failure should the final system not meet the noise objective. (The TD-2 system fell a little short of its noise objective, once fully utilized; further minor improvements are possible, moreover, even after a system is in operation, and such improvements were underway for TD-2 at the time of writing). The attitude toward the three goals might be summarized as follows: it seemed likely that for a reasonable development effort a system

\footnotetext{
${ }^{13}$ More precisely, a minimum signal-to-noise ratio at hours of peak system use under average atmospheric conditions.

${ }_{14}$ The goal of 1,800 telephone conversations per channel was reasonable, given the base-band width-10 mc-of the AM signal whose information content is transmitted over a channel. The $10 \mathrm{mc}$ width was largely dictated by the existing equipment (developed for a coaxial-cable long-haul system) which could combine telephone conversations (1,800 of them, roughly) into an AM signal whose width was a bit less than $10 \mathrm{mc}$. The choice of $10 \mathrm{mc}$ was also influenced by the possibility of a future demand for transmission of theater television, which would require a base-band of this width.

${ }^{25}$ It will be recalled that for each two-way broad-band channel there is, at each repeater station, a distinct pair of receivers and a distinct pair of transmitters.
} 
could be obtained whose production cost was acceptable, whose noise came close to the noise objective, and whose message capacity and reliability were acceptably near the respective objectives (but further than the system's noise is from the noise objective). Development effort and production cost were to be increased, as became necessary, to reach acceptable values of the three performance magnitudes. Should a point in development be reached where an acceptable value of one of them could not be attained without a drastic increase in development effort or production cost, a decision whether or not to sacrifice performance significantly would be required. But that would be a bridge to be crossed when and if it appeared: there was no need to formulate beforehand a strategy for decision in the many conceivable contingencies of this sort.

No estimate of the total TH development effort was contained in the prospectus which, in 1952, accompanied official laboratory authorization of preliminary system planning. The prospectus did make a prediction about production cost, namely that the first cost of $\mathrm{TH}$ per channel-mile (using the existing TD-2 towers) would be about $\$ 1.25$ less than for TD-2. (This prediction turned out, as we shall see, to be a moderate underestimate.)

Generating Alternative Major System Characteristics Consistent With the Objectives

The objectives having been formulated, many decisions remained as to certain important system characteristics. By a system characteristic we mean either (1) a number (or set of numbers) that characterizes the system (rather than any one component) but is distinct from the magnitudes defining the objectives; or (2) the specification which of several different ways of attaining the objectives is to be used (e.g., which of two possible, qualitatively different major components is to be used to perform a certain function). Alternative sets of characteristics consistent with the objectives were found, and choice among these sets was subsequently to be made. It was important to generate some alternatives at this stage, since uncertainty as to the effort required to realize any given set of characteristics was great. To formulate any set, however, is a costly analytical job, and many possible alternatives were never presented. ${ }^{16}$

\footnotetext{
${ }^{10}$ The final choice of system characteristics may reflect what system planners are fond of calling a "design philosophy"-i.e., a general approach to a wide class of problems
} 
The most important characteristic was the frequency allocation plan. Two alternative plans were developed in 1952. One was the twofrequency plan finally adopted (a given signal traveling in a given direction uses two frequency bands, shifting from one to the other at each repeater station). This would allow, within the available $500 \mathrm{mc}$ space, eight two-way broad-band channels and $52 \mathrm{mc}$ of guard space. The other plan was a four-frequency plan wherein each two-way broadband channel uses a separate pair of frequencies for each direction of transmission. Adjacent bands would be allowed to overlap somewhat in the latter plan but, even so, only six two-way broad-band channels could be fitted into the $500 \mathrm{mc}$ space. ${ }^{17}$ The first plan was therefore preferable, but its feasibility depended on further advances in the design of the antennas and the associated microwave filters. The difficulty of these advances was very much in doubt.

Another important pair of alternatives presented in 1952 were two repeater types. One possibility was an i-f type in which gain, regulation, and frequency shift are accomplished after bringing the signal down to i-f. ${ }^{18}$ The other possibility was an all-microwave type where modifications of the signal are accomplished without bringing it down to i-f. The former type is necessary in any case at main repeater stations where channel switching ${ }^{19}$ is required, but a choice between the types had to be made for all other repeater stations.

A third major pair of alternatives was to use or not use a low-noise traveling wave tube in the receivers.

A fourth very important set of alternatives consisted of the possible allocation of acceptable noise among major parts of the system so as to meet the total noise objective for telephone message and TV

posed by the system. In TH, for example, such a philosophy opposed the use of "mopup" circuits following a component to correct undesirable distortions generated by the component; the component was to be designed, in general, so as to make mopping up unnecessary.

${ }^{17}$ In both plans the width of each one-way channel is $28 \mathrm{mc}$. Given the width of the AM base band other widths could have been explored (permitting more or fewer channels, with a penalty or gain in signal-noise ratios). But developing each alternative plan and exploring its consequences is expensive; it seemed reasonable to confine the comparison between two and four-frequency plans to the $28 \mathrm{mc}$ width. In any case, $28 \mathrm{mc}$ was regarded as a good bet for a width for which near-attainment of the noise and capacity objectives would be a reasonable development task.

${ }^{18}$ The precise intermediate frequency had not yet been chosen.

${ }^{19}$ I.e., switching a group of telephone conversations or a television broadcast from one specific two-way broad-band channel to another. This occurs at stations from which branches off the main trunk routes originate. Switching to a protection channel is a second kind of switching. Provision for either or both kinds is needed at two out of three stations. 
transmission. A tentative "target" allocation was chosen in 1952. The acceptable noise contribution of central offices, terminals, and repeaters was specified. It "added up" to the total noise objective. ${ }^{20}$ It was explicitly recognized that deviations from the target allocation would be permitted as they arose; if it turned out to be difficult not to exceed target noise for one source, the excess would be allowed, provided the noise in another source could be made to fall short of the target there by a roughly compensating amount.

Finally, a target requirement was set for system equalization. This magnitude measures, to put it crudely, the variation, as between a given channel's original amplitude-modulated base-band frequencies fed into a link of the system, of the strengths with which they emerge at the FM-AM terminal at the other end of the link, as well as their variation with respect to phase at the other end of the link. It is an important determinant of each channel's message capacity, given a maximum noise level, and it depends on the performance of a number of components. Some variation from the target value, initially set the same for each channel, was to be admitted.

Note that the system characteristics are closely interdependent: given the objectives to be met, and given all but one, the admissible values of the remaining characteristic are sharply constrained.

\section{Broad Requirements for Major Components}

The final planning task to get underway in 1952 was the formulation of broad requirements for major components consistent with each other, with the objectives, and with some of the alternative sets of system characteristics.

ANTENNAS. In the preferred two-frequency plan a repeater station $\mathrm{N}$ receiving a desired frequency from repeater station $\mathrm{N}+1$ will also receive interfering signals from repeaters $\mathbf{N}-1$ and $\mathrm{N}+1$. The only known. way of discriminating against this interference is to improve antenna front-to-back ratios (very roughly the ratio of the antenna's efficiency in receiving or radiating

${ }^{20}$ The rule for totalling the contributions is more complex than simple addition. In addition to breaking down total noise into contributions of these three parts of the system it was also broken down into "types" of noise (fluctuation, intermodulation, and crosstalk noise). A given component may contribute one type of noise but not the others; the types are equally important to the total system noise. It goes without saying that attainment of a noise level for a part of the system below the target level for that part is desirable if it can be achieved with no additional effort. 
energy in the desired direction to its efficiency in doing so in the reverse direction). Minimum requirements for antenna front-toback ratios were computed such that, under the two-frequency arrangement, the "target" for each repeater station's contribution to system noise would be met-provided that reasonable requirements on other components are met.

TRAVELING WAVE TUBE AMPLIFIER. It was concluded that to guard against fading (which can periodically occur between any two repeater stations), while meeting the noise target for repeater stations and the message capacity goal for channels, each repeater should be capable of providing up to $100 \mathrm{db}$ of gain and its traveling wave tube amplifier should have a power output of "about" five watts.

Requirements for the low-noise tube, on which development work was under way, were also formulated in case the alternative of using one should be adopted.

OTHER REPEATER COMPONENTS. Requirements on other major components of both i-f repeaters and all-microwave repeaters were roughly sketched. Some of these components were to be adaptations of TD-2 components, but others (e.g., the common microwave generator, the i-f amplifier) would require substantial development effort.

POWER SUPPLIES. Voltage requirements for each repeater station's power supply were formulated to meet the needs of the traveling wave tubes and other repeater components.

ALARM AND CONTROL CIRCUITS AND AUTOMATIC SWITCHING. A description was given of proposed alarm and control circuits, together with devices for automatic switching to protection channels. These features promised to meet the objectives for system reliability.

To summarize: The requirements drawn up for major components in this period were very broad and not rigid. They consisted generally of upper and lower limits to the main component characteristics. But they were consistent with each other, with the system objectives, and with some of the alternative sets of system characteristics. It could be stated with great confidence that, if major components meeting the requirements were developed, then the system objectives would be met. While there was considerable uncertainty about how difficult attainment of any requirement would turn out to be, the 
requirements seemed reasonable guides for the next stage of development. It was quite clear, however, that should a given requirement prove difficult to meet, another one might be substituted, perhaps a requirement consistent with different sets of system characteristics among the alternative sets previously generated, or even with different objectives, implying quite new system characteristics.

\section{3-54: CHOICE BETWEEN ALTERNATIVE MAJOR SYSTEM CHARACTERISTICS, FIRST DETAILED SYSTEM PLANNING, INITIAL COMPONENT DEVELOPMENT, AND DEVELOPMENT SCHEDULING}

During the next stage in the development of TH, choice between major alternative system characteristics was made; the system was planned in detail for the first time (including requirements for a number of minor components); the development of major and minor components got well under way; and target dates for the main events in development were set. Finally, a tentative development budget was drawn up and allocated. All these activities overlapped in time.

\section{Choice Between Alternative Major System Characteristics}

Choice between alternative sets of major system characteristics was made possible in this stage because enough evidence had accumulated about the development difficulties implied by each. The evidence consisted of observations on experimental models of the key components which particular alternatives would require as well as further paper analysis of the requirements implied and the difficulty of attaining them.

LOW-NOISE TRAVELING WAVE TUBE. A decision not to use a low-noise tube in the receivers was made early in 1954. The noise figure obtained in some experimental tubes was not so low as had been hoped and at the same time the converter itself (using a newly developed crystal) promised a much better noise figure than anticipated earlier. Hence the advantage of incorporating a low-noise tube ahead of the converter appeared to be quite small and not worth additional development effort.

FREQUENCY PLAN. The two-frequency alternative was chosen. As a result of experimental work with antennas it appeared very likely that an antenna design yielding the required front-to-back ratios could be obtained. The precise spacing of channels was not decided upon until the end of 1954. (Given the channel width 
of $28 \mathrm{mc}$, previously chosen, it depended essentially on the width of the guard space between the two groups of eight one-way channels.)

REPEATERS (OTHER THAN MAIN REPEATERS): I-F VERSUS ALLMICROWAVE. The possibility of all-microwave repeaters was rejected. The principal reasons were (1) difficulties discovered in achieving frequency shift in such repeaters, and (2) the increasingly likely prospect that, as the microwave network becomes denser, virtually all stations will become nodes of the network and will have to be provided with a channel switching ability.

NOISE ALLOCATION. By mid-1954 the noise objective had been slightly relaxed from that of two years earlier and the planned noise allocation had accordingly been changed slightly from the earlier target allocation. Now, moreover, it was given in somewhat greater detail. The target noise from repeater stations, in particular, was broken down into subtargets, corresponding to several repeater components. Early design work on noise-critical repeater components, undertaken in the intervening two years, had made this breakdown possible.

SYSTEM EQUALIZATION. A small relaxation from the original target was decided upon for certain specific channels and a slightly more stringent target was set for any channel carrying color television. The difficulty of achieving the revised targets was still very uncertain at the end of 1954 , since it depended on the performance of a number of undeveloped components.

\section{First Detailed System Planning}

The end products of this period's detailed system planning were block diagrams of the system. They showed the path of the signals from component to component as well as specifications (requirements) for the components, stated in greater detail than before. This required choice of some minor system characteristics not dealt with before.

\section{More Detailed Component Requirements}

The choice of a major system characteristic was generally accompanied, as we have seen, by a more detailed statement of the alternative chosen. Similarly, requirements for major components, consistent with the chosen alternatives, were now formulated in considerably greater detail, involving in some cases specifications to be met by many of the smaller parts making up a major component. In addition, 
requirements were generated for minor components, not bothered with in the previous stage of development. The level of detail, however, was quite uneven - high for some (major or minor) components, low for others. This occurred for two main reasons; we shall discuss each in turn.

VARIATION IN DEVELOPMENT UNCERTAINTY OF COMPONENTS. The greater the uncertainty as to size of development effort needed to meet given requirements for a component, the less detailed, in general, were (1) the requirements formulated for it in this period, and (2) the requirements formulated for other components whose performance closely depended on it. Requirements for the components closely dependent on a highly uncertain component were stated broadly to provide consistency with a wide range of possible specifications for the component in question.

Consider, for example, the isolator and the common microwave generator. The isolator developed was a novel solution to the problem of blocking reflections from the antenna before they reach a repeater to which the antenna is connected. That the material (a ferrite) and the design would function properly became evident in 1953. Hence, in mid-1954, a precise minimum level of attenuation of the undesired antenna reflections was specified, as well as a maximum acceptable forward loss (i.e., loss of strength in the signal that passes through the isolator in the desired direction). In formulating those component requirements whose soundness depended on isolator performance, there was then no need to "hedge" to allow for alternative levels of isolator performance.

The common microwave generator, on the other hand, was a much more problematic component. Its critical property was reliability. The alternative to a common generator at each repeater station was a set of generators, one for each channel (this alternative was used in TD-2). The common generator's chief advantage is that it greatly reduces the number of tubes and other unreliable elements required for microwave generation (but requires a greater number of "passive" elements). Consequently the probability of failure of any single channel due to failure of its microwave supply at some repeater station is greatly reduced. The probability of failure of all channels, however, is somewhat increased, even with the use of a stand-by common generator at each repeater. The difficulty of reducing the latter probability to negligible levels was quite uncertain, and no requirement for the probability was decided upon in this stage of 
development. The many possible ways of generating the needed family of microwave frequencies ${ }^{21}$ implied different generator reliabilities and a sufficiently wide exploration of these alternatives was far from complete at the end of 1954. In particular, a judicious choice of the i-f would make it possible to require only a small number of basic oscillator frequencies. Combinations of these could then be found which, when impressed on the i-f in the transmitters, yield the required family of 16 microwave carrier frequencies. The fewer the basic oscillator frequencies the common microwave generator is required to produce, the simpler and more reliable is the generator. The judicious choice of i-f was not made, however, until the end of 1954. The possibility of separate-channel generators was, in fact, still held in reserve until then.

VARIATION IN SYSTEM CRITICALITY OF COMPONENTS. Quite aside from having very uncertain development difficulties, a component may be highly "system critical"; its performance affects the appropriateness of a great many other components. In principle (though this probably rarely occurs in practice) a component could display virtual certainty about its development difficulties and yet be highly system critical. It was natural, in $\mathrm{TH}$, to be particularly careful about prematurely issuing detailed requirements for highly critical components, even if their development difficulties were only moderately uncertain.

Choosing, at a given point in development, the appropriate level of detail for a critical component's requirements (which are taken as predictions about the component's final properties when planning other components) calls for a difficult balancing of two costs. A balance must be struck between the risk of having to revise the entire system when detailed predictions about the component turn out to be wrong, and the cost in total system development time of waiting to announce predictions (requirements) until this risk is small (because the component is already well into development). Since much depends on a critical component, planning and development cannot proceed without some prediction as to its final performance; but if too detailed predictions are taken too seriously too soon, costly revisions of the entire system are likely to be needed.

The isolator is a good example of a component which was relatively uncritical in the above sense. The power supply was an example of a critical component whose development uncertainties were moderate.

${ }^{21}$ The frequencies needed were precisely determined once the two-frequency plan had been decided on and a division of the $500 \mathrm{mc}$ band into channels and guard space chosen. 
The requirements on the power supply at the end of 1954 were still very broad, not so much because its development was a puzzle but because too much else depended on its precise performance.

\section{Generation and Choice of Minor System Characteristics}

Part of the process of formulating more detailed component requirements was the generation of alternative minor system characteristics -magnitudes which are not attributes of only a single component and which it was premature to consider in the previous stage of development. Among such magnitudes were, for example, the intermediate frequency and the channel guard space. The proposed alternative characteristics had to be consistent with the chosen values of the major characteristics. To formulate final component requirements, choice among the alternatives had to be made, though choice could in some cases be deferred until development of the dependent components was already under way. (Design work on the i-f amplifier, to meet certain requirements that did not depend on frequency, could and did precede final choice of the i-f.)

\section{Initial Component Development}

Requirements for a number of important components having been issued in 1953-54, the developers of these components had goals, though they varied widely in precision. By the end of 1954, designs of all major components had been proposed, and in several cases hardware had been constructed. We shall discuss the development of the principal components in the next section.

\section{Scheduling Development and Allocating Manpower}

In mid-1954 a schedule for development activities was released. For most components it showed a date for each of four events: completion of preliminary requirements (already attained in many cases); completion of a preliminary model (also known as a "breadboard model"); completion of a laboratory model (also known as a "brassboard model"); and release of manufacturing information. The fourth event was to occur by 1957 for all components. The distinction between the two kinds of models is difficult to characterize in a general way. For each component, the two models serve as checkpointstheir completion is an opportunity for a reassessment and, if this is worthwhile, a revision of the component's specifications (a last-chance opportunity, in the case of the laboratory model). Completion of each 
model for a component probably marks a sharp jump in knowledge about the components, i.e., in the accuracy of predictions about additional effort required to meet given specifications.

The schedule was a goal, in the same sense as component requirements were, but was probably regarded as still more likely to undergo later changes. At the same time rough estimates were made of the sequence of annual man-years (of technical personnel) needed to achieve the schedule, and the allocation of manpower between major tasks in each year. The total manpower estimates were: 53 technical man-years ${ }^{22}$ in 1954, 62 in 1955 and 1956, and 59 in 1957.

\section{5-57: FULL-SCALE COMPONENT DEVELOPMENT AND}

\section{FINAL SYSTEM PLANNING}

By 1955 full-scale development of all components was underway. In 1955-57 the final system plan was worked out in detail. Even the most broadly stated component requirements issued in the preceding period were not immutable. Part of the task of a component's developers was to be continually alert to the possibility that a reasonable modification of the requirements might make the task of completing the components significantly easier and to propose such modifications. In developing some components, parallel exploration of alternative designs, all holding promise of meeting the (tentative) requirements, was undertaken.

We shall discuss most of the major components, but not all. The periods considered overlap the period treated in the preceding section.

\section{Traveling-Wave-Tube Amplifier}

While this was probably the most difficult component to devel$o p$, and one of the most system critical, it was, as we have seen above, one of the earliest to be started. The early experimental models of the traveling-wave tube operated at the wrong frequency and had many defects. In April 1953 experimental 6,000 $\mathrm{mc} / \mathrm{sec}$ tubes became available, together with most of the required items of $6,000 \mathrm{mc} / \mathrm{sec}$ test equipment, but many difficulties remained. There were three major interrelated problem areas: the "matching" ${ }^{23}$ of the waveguide (through which the signals to be amplified enter the tube) to the helix; the design of a suitable

${ }^{22}$ In obtaining these figures, a man-year for a technical assistant is counted as half of a man-year for a member of the technical staff.

${ }^{23}$ With respect to impedance. 
magnetic focusing structure surrounding the tube; and the coupling of the amplifier to certain adjacent circuits. The requirements at which work in these three areas aimed were broadly stated through 1955. The goal was an amplifier of at least $25 \mathrm{db}$ gain with a power output of about five watts ${ }^{24}$ requiring very little adjustment and with noise characteristics and other properties "satisfactory."

By the end of 1953 two important but relatively easy decisions had been rather firmly made: the critical properties of the power supply for the tube had been chosen; and it had been decided to incorporate a limiter ahead of the tube so as to remove residual amplitude variations that would be turned into phase variations by the tube. This largely left the first two of the above three problem areas.

Much of the work on helix-waveguide matching had to await the availability, in mid-1954, of a crucial item of $6,000 \mathrm{mc} / \mathrm{sec}$ test equipment (a frequency generator for the entire $500 \mathrm{mc}$ band). This permitted extensive experiments on various tube and waveguide designs; their general aim was wide-band matching (over the entire $500 \mathrm{mc}$ band), which would make it possible to tune (adjust) the amplifier in the same way for all channels and to replace tubes without retuning. This was a reaction to the TD-2 situation where separate tuning was required for each channel and maintenance was complex. It was an early design goal for the amplifier, but it was not a formally stated requirement, on whose performance specification of many other parts of the system depended. After many trials (including experiments with the leading in and out of signals by coaxial cables and not by waveguide at all), a waveguide and tube design was attained (about the end of 1955) which achieved wide-band matching and, in combination with the magnetic focusing structure that had by then been developed, provided the gain aimed for at acceptable noise levels. ${ }^{25}$

The work on magnetic focusing structures was aimed at highly accurate focusing of the electron beam ${ }^{26}$ by an easily maintainable

${ }^{24}$ Only the power output goal had been stated in 1952.

${ }^{25}$ I.e., at noise levels such that the goal for repeater noise announced in mid-1954 could be met, given the noise properties which now promised to characterize the other repeater components (most of which were nearly developed).

${ }^{28}$ I.e., at a longitudinal magnetic field parallel to the tube which is very strong relative to the cross field (perpendicular to the tube), the ratio of the two strengths being very precisely determined. 
structure of reasonable weight; the focusing structure of the earliest experimental amplifiers had been very heavy and had required complex adjustment. The design tested up to the end of 1955 had proved unsatisfactory, and it became clear that the difficulty of the magnetic structure problem had been seriously underestimated. The requirements the magnetic field must satisfy in order to achieve the broad amplifier goals were still not well understood, and magnet designs meeting given field requirements were unexpectedly difficult to achieve. At several points measuring equipment not previously planned turned out to be essential and had to be quickly developed. The magnetic structure had to be shielded; a way had to be found to prevent the powerful field of the magnet from interfering with components adjacent to the traveling-wave-tube amplifier without disturbing the delicate focusing of the traveling-wave-tube's electron beam. A new approach to magnetic structure design was tried early in 1956-periodic-field focusing rather than the previous straightfield method. The hope was that the new approach might permit a smaller and more easily designed magnet assembly. The two approaches were pursued in parallel throughout 1956; periodic focusing was finally abandoned as too difficult.

Early in 1957 an amplifier design had been obtained achieving sufficiently accurate wide-band matching and beam focusing to attain the broad amplifier performance goals with a satisfactorily low maintenance effort.

To summarize, the traveling-wave-tube amplifier was critical to the planned system and highly uncertain as to development difficulties. It was itself a system, composed of several major and several minor components for each of which feasible requirements consistent with each other and with the broadly stated amplifier goals had to be found. The first requirements decided on were those most certain of attainment and least critical with respect to other requirements on amplifier components (power needs, properties of the limiter to be used ahead of the amplifier, cooling mechanism for the tube). In developing all major parts of the amplifier, alternative approaches were tried, some simultaneously, some successively.

The development of the amplifier ran into major unexpected difficulties. But the amplifier was so critical to the system-in the stage of development the system had already reached when these difficulties 
came to light-that the effort needed to resolve these difficulties appeared well worthwhile. An alternative might have been a major downward revision of the amplifier goals or the substitution of an amplifier not based on the traveling wave tube. Such a change would have required a major revision of the system and its development schedule, at a cost far outweighing the expected effort of resolving the traveling-wave-tube amplifier's difficulties. In retrospect, it might appear that a greater exploratory effort on magnetic focusing structures should have preceded any formulation of amplifier goals for use in planning the rest of the system. But it may be that the strategy pursued was generally sound (that it is a strategy the laboratory is content to pursue, in the light of experience, in all such situations). For it may be that the balance between the risk of system revision and the rewards of early planning of a critical component had been reasonably struck, and that the unexpected difficulties were improbable misfortunes. ${ }^{27}$

\section{Shorter Notes on Some Other Components}

I-F AMPLIFIER. Early work on a high figure-of-merit tube for the i-f amplifier had been undertaken in 1953 and was well under way even before the amplifier's critical requirements had been broadly formulated. Such a tube (which provides the amplifier with a high signal-to-noise ratio) would probably be useful in fulfilling any performance goals. Requirements had to await the decision as to i-f frequency and the allocation of the $500 \mathrm{mc}$ band to broad-band channels. By mid-1954 goals for the i-f amplifier included the frequency band to be amplified, ${ }^{28}$ the amplifier's contribution to total repeater noise, its gain, and its distortion characteristics. It.was decided to design the amplifier so as to minimize the dependence of distortion on tube characteristics; this would avoid the tendency of the TD-2 system's i-f amplifier towards variation of distortion from repeater to repeater (whose amplifier tubes differ in age). The goals were, of course, not rigid. They were moderately critical with respect to goals for other parts of the system and were moderately uncertain of attainment.

Several important alternative design approaches were pursued in developing the amplifier. The amplifier tube first adopted

${ }^{27}$ An organizational interpretation of the difficulties that arose with respect to the magnetic focusing structure is given in a later section dealing with functional departments.

${ }^{28} \mathrm{~A}$ band $28 \mathrm{mc}$ wide around the chosen $\mathrm{i}-\mathrm{f}$ of $74.1 \mathrm{mc} / \mathrm{sec}$. The width of $28 \mathrm{mc}$ for each broad-band (microwave) channel had been chosen in 1952. See footnote 17, above. 
turned out to have a cathode prone to warping and an amplifier design using a lower-performance tube and more stages was substituted. Two kinds of gain control-thermistor and grid-were explored, in part simultaneously, and the latter adopted. Two kinds of circuitry were explored in sequence: an attempt at printed circuitry proved very difficult and was dropped, in mid-1955, in favor of more conventional circuitry.

CONVERTER. Design of this component was finished early in 1954 , subject to satisfactory completion of a crystal then still under development. The noise goal proposed (in 1952) for a converter preceded by a low-noise tube would then be more than met, and so the low-noise tube was dropped. Subsequent system planning essentially took converter performance as given, and the missing crystal became available, meeting expectations, in 1955.

POWER SUPPLY. Requirements for this component were chosen quite late - in 1955. They depended on those properties of many other components which determined the power needs of those components. Designing a required power source was a straightforward task. In formulating the power requirements, however, it was possible, to some extent, to simplify design of the powerusing circuits by requiring the supply of a large variety of voltages. On the other hand, voltage requirements could be standardized, but this would require more complex using circuits. After preliminary study and debate, an approach in between the extremes of no voltage standardization and complete standardization was adopted.

FM TERMINALS, ENTRANCE LINKS, ALARM AND CONTROL CIRCUITS. Requirements for these components, like requirements for the power supply, were highly dependent on the system but were not uncertain with respect to development difficulty. Requirements for them were not chosen until well into 1955, and development was completed in 1956 and early 1957. Since the size of the development effort for these components was accurately predictable and goals for them could not be accurately specified until the system was well under way, the postponement of their development was reasonable.

\section{Final System Planning and the Completion of System Development}

By the middle of 1958 the block diagrams of the system had taken their final form and the component specifications had 
been completely filled in. Manufacturıng information for nearly all components had been released and the remainder would shortly follow. The system displayed only very minor departures from the original system objectives. The system's production and operating costs will also be close, it appears, to predictions made in 1954.

With respect to the development schedule presented in 1954, there had been "slippage" for some components (particularly the travelingwave-tube amplifier), but none of it much more than six months. The 1954 prediction as to manpower (and hence development cost), had, however, proven substantially optimistic. The actual total technical manpower used was roughly as follows: 56 man-years in 1954 (55 were predicted); 72 man-years in 1955 (62 predicted); 90 in 1956 (62 predicted); 88 in 1957 (59 predicted); and 95 in 1958. It would appear that the goal for total development effort was much more willingly sacrificed than the development schedule, and the latter more willingly modified than the objectives and the production and operating costs. The unplanned increase in development effort, moreover, seems to have been sufficient to "absorb" the system's unexpected difficulties, since the other goals did not need to be significantly relaxed.

\section{Organization of TH Development}

\section{SYSTEMS ENGINEERING}

Of the three major departments of the Bell Telephone LaboratoriesResearch, Development, and Systems Engineering-the latter two played a part in developing TH: The general function of the Systems Engineering Department is continually to explore the needs of the Laboratories' customers-the operating telephone companies-while at the same time keeping closely in touch with the technical possibilities for meeting these needs. From time to time this function culminates in a formal proposal, described in a prospectus, for a specific system to be developed. In consultation with the Systenıs Engineering Department and the Laboratories' management, A. T. and T. (the principal prospective user of the system) and Western Electric (its manufacturer) decide whether or not the Laboratories should be authorized to develop the proposed system. This is an economic 
decision, making use of forecasts in the prospectus about development time and cost, production and operating costs, and system performance. Generally, the forecasts as to development time and cost are the most cautiously worded and are violated with least regret. It is deeply understood by all parties to the decision that the final system performance and production and operating costs corresponding to a given development effort are initially uncertain.

In the case of $\mathrm{TH}$, two or three persons from Systems Engineering prepared the first prospectus (in 1952), based on preliminary studies performed by personnel of Development. The prospectus estimated future demand for long-haul service and the operating savings which could be expected from $\mathrm{TH}$, using existing TD-2 routes, as compared with building new TD-2 routes. After the prospectus appeared, the management of the Laboratories, A. T. and T., and Western Electric authorized initial development at a low level of intensity for two years (an upper limit on the technical man-years to be used was, in effect, imposed). In 1954 a second prospectus appeared. It made much more specific predictions, in the light of the work performed (in the Development Department) in the intervening years, about the final system and the development effort required. The development schedule described above was included. The same authorities who had approved the 1952 commitment now authorized completion of the TH development; they considered it sufficiently likely that the objectives described in the new prospectus could be closely approached for the predicted development time and cost. Some deviation from the latter, i.e., from the approved annual manpower targets (increasing over the project's remaining years), would be acceptable.

It is largely within the Development Department of the Laboratories that TH was developed and it is the organization of the task within that Department that we shall examine.

\section{ORGANIZATION OF TH WITHIN THE DEVELOPMENT DEPARTMENT}

Two broad classes of engineers were engaged in developing $\mathrm{TH}$ - the planners of the system and the developers of its components. ${ }^{29} \mathrm{~A}$ TH System Analysis Group composed of planners was created in 1952, at the origin of the system, and maintained throughout its development. Its early function was to generate major system characteristics and rough block diagrams (showing the parts of the system and what

${ }^{29}$ Some individuals, of course, belonged to both classes. 
each "does" to the signal transmitted, as well as broad specifications for major components). Its continuing task was to modify all of these and make them more detailed, as new knowledge appeared. The size of the group stayed close to five persons from 1954 on.

In contrast with the systems analysis group, the identity of some of the component groups shifts and fades through the years. Personnel switch back and forth between related components and some component groups appear to merge completely. This reflects not only the arbitrariness of any division of the system into components, but also a conscious policy, to be detailed below, of assigning broad component goals and encouraging the freest communication (and, to some extent, the making of agreements) between related groups.

The systems analysis group's role was not that of central decision making authority, issuing instructions to the component groups. Formal authority rested with what we may call an executive group of two or three individuals - the project engineer, the head of the section (Transmissions Systems) of the Development Department in which the bulk of $\mathrm{TH}$ effort took place, and (though he seldom. exercised authority) the head of Development or his deputy.

\section{The Organizational Ideal for $\mathrm{TH}$}

An ideal pattern, at which the formally designated leaders of TH seemed consciously to have aimed, was roughly as follows.

The systems analysis group formulates alternative major system characteristics consistent with the objectives ${ }^{30}$ (which were formally issued by the Systems Engineering Department after preliminary consultation with the future developers of the system). It deduces from these a system plan, i.e., block diagrams with tentative requirements for the system's major components, consistent with one or more of the alternative sets of system characteristics and reasonable in the light of the information then available about the difficulty of attaining given requircments. The executive group approves these requiremonts as trial goals that are good "guesses" at the final ones, and tentatively assigns personncl to the component groups. The component groups start working toward the tentative requirements.

As soon as a component group is able to make a more confident estimate of the effort needed to meet the requirements it so informs

${ }^{30}$ The objectives themselves may later be changed, in accordance with the "trade-off" possibilities mentioned earlier; in the case of $\mathrm{TH}$, as we have seen, this did not happen to a significant extent. 
the executive group. It is, moreover, not only permitted but actively encouraged to tell the executive group as soon as it has evidence that a minor modification of its requirements (or a joint modification of its requirements and those of a related component group) would significantly ease its task (or the joint task of the two groups). This information from the component groups forms the basis for a series of "parliamentary" conferences, presided over by the executive group, with members of the component groups and the systems analysis group as participants. The conferences achieve a consensus as to the requirements for which modification should be attempted, as to a new tentative allocation of manpower ${ }^{31}$ among groups and over time, and as to a tentative time schedule for completion of successive stages in each group's task.

The systems analysis group then works out the proposed modification of the system plan; the modification may call for replacing some of the system characteristics of the first plan with previously generated alternatives. Accompanying the modification, there is a greater detailing of major component requirements and the introduction of minor requirements. Both may be further modified later on, requiring, in turn, a further modification of the system plan and perhaps a further reallocation of manpower and a revision of schedules. The further changes are again settled in parliamentary conferences.

At all stages the executive group is easily accessible to the component groups. In the traditional informality of the Development Department (housed in a single building), conversations (face-toface or by telephone) are generally as easy to arrange between members of different groups as between members of the same group. The members of the executive group, moreover, keep very well informed about the activities of the component and systems analysis groups, becoming, on occasion, part of certain of these groups working directly with them, for example, in compiling the evidence in favor of modifying their requirements. The executives might better be thought of, in fact, as central storers of information than as makers of decisions. Their knowledge of the status of all parts of the system is essential to decisions about changes in requirements, manpower allocations, and schedules, but the decisions are collectively made by the members of the parliamentary conferences (though formally

${ }^{31}$ That is, an allocation of the annual total manpower availabilities (increasing over the life of the project) approved by the laboratory management. These availabilities are "targets," which may later be revised if the system's progress should be slower or faster than anticipated. 
issued, in memoranda or other documents, in the name of an executive).

Each component group is free to pursue its own ideas about the internal design of its component, exploring parallel approaches (or several approaches in sequence) if it wishes.

Finally, in the ideal organizational pattern, the relations between groups working on related components are very close. Each group can easily inform itself (and is kept informed through generally distributed memoranda) of the work of a related group; it does not need to proceed through the executive group in acquiring such information. Two related groups are encouraged to explore together designs for the two related components which would lessen their joint effort in meeting their (tentative) assigned goals, as well as to propose mutually compensating changes in these goals that would leave the system no worse off.

Among the better documented component developments in which the ideal organization pattern was closely approximated, several stand out. (1) Once the basic requirements for the common microwave generator-the frequencies it was to generate-were decided on, there remained a number of other requirements, which were tentatively chosen. The most troublesome one dealt with unwanted "tone products" (extraneous frequencies). A series of memos and minutes of conferences reveals the process of refining the requirement on tone products to obtain one that reasonable effort can achieve and the process of adjusting the system plan accordingly. (2) There is a similar process with respect to the more detailed requirements given the antenna design group. (3) There are memoranda from the executive group to the test equipment group documenting the ordering of test equipment and containing sentences such as this: "Any of these requirements that appear unduly difficult should be brought to our attention for possible modification." (4) The i-f amplifier group explored, as we saw, two major circuitry approaches. The idea for the first approach and the decision to drop it and pursue the second originated within the group.

There were, of course, continual minor exceptions to the ideal pattern-minor requirement modifications and minor reallocations of manpower, which appear in retrospect desirable, were thought of by component groups but never acted upon. 
Functional Departments: the Major Exception to the "Ideal" Pattern

The major exceptions to the ideal pattern occurred with respect to some (but not all) of the functional departments (within the Development Department) that worked on TH. These are departments that perform specialized development tasks for all systems under development. The principal ones, in the case of TH, were the tube department, the magnetic devices department, the solid-state devices department (which developed the converter crystal), and the testequipment department. During the TH development, these departments had to work on components for other systems than TH. The allocation of such a department's manpower to TH work, given the competing needs of other systems and the current total manpower targets for $\mathrm{TH}$, was generally settled by negotiation between the head of the functional department and the TH executive group. Occasionally, one of the two or three executives of the Development Department was called upon to arbitrate. The solution sometimes involved assignment of TH personnel from outside the functional department to work with the functional department so that the total TH manpower target was not exceeded and the functional department was able to meet its competing needs.

Communication between the members of the functional departments working on $\mathrm{TH}$ and the groups working exclusively on $\mathrm{TH}$, and vice versa, seems to have been, on significant occasions, more difficult than communication between the latter groups. This seems to have been because, in the first place, consultations on matters that held implications for the reallocation of manpower within the functional department often had to involve the head of the functional department - to whom its TH workers were responsible and to be arranged through him. In the second place, a casual survey suggests that members of the functional department had their own internal loyalties and interests; they may sometimes have been motivated less by the specific desire to make TH work well than by the desire to perfect the devices which were their specialty independently of this particular using system. Communication by a functional department's TH workers of tentative notions for improving the component they were developing in the specific context of TH may have been less spontaneous and frequent than was true of the other TH groups.

Functional departments exist in a laboratory, however, because of 
the advantages they possess. There are distinct economies of scale in combining all development work on certain devices, versions of which are required again and again by many systems, into one development group. Moreover, it is an important Bell Laboratories policy to prepare for the systems of the future by developing and placing "on the shelf" laboratory models of components with new and interesting properties. This is a major responsibility of most of the functional departments. Whether these advantages outweigh the difficulties that some system projects experience in dealing with those departments is an important question, on which a single case study can shed no light.

In the history of $\mathrm{TH}$ there are several fairly well documented instances of major exceptions to the ideal organizational pattern resulting from a functional department's participation in the system project. The best documented instance centers around the magnetic focusing structure of the traveling-wave-tube amplifier. As we have seen, the task of developing an acceptable structure proved to be unexpectedly difficult. The problem had been entrusted to a functional department - a magnetic devices department which was simultaneously assisting other system projects. At the same time, developing the traveling-wave tube itself was primarily the responsibility of a group within another functional department - the tube development department. The magnet difficulties, when they arose, were partially attributed by the TH executives to insufficient allocation of manpower to magnetics and to poor coordination between the magnetics and tube groups (for example, the problem of physically mounting the magnetic structure on to the tube had been given insufficient attention, according to the TH executives). The TH executives were coming to feel that the magnetics work should be done not within a functional department but rather by a group set up to work exclusively on $\mathrm{TH}$.

A series of conferences took place, attended by the TH executives, the heads of the two functional departments, and the principal developing engineers concerned. The outcome was as follows: magnetic structure work was to continue as a permanent functional responsibility and the $\mathrm{TH}$ project was to have its own temporary magnetics group (for which new manpower was authorized ${ }^{32}$ ), independent of the functional group. It was the TH magnetics group that

\footnotetext{
${ }^{32}$ The additional manpower would eventually show up as a small permissible increase in total target manpower unless compensated for by decreased use of manpower in other parts of the system.
} 
developed the structure finally used in TH. The minutes of the final conference make the case for preserving the general functional responsibility thus:

In considering the assignment of responsibility the following factors need to be weighed. The magnetic structure is an intricate and highly specialized kind of unit. It is an entity which has well defined natural boundaries. Design as well as exploratory work calls for highly specialized skills, which usually take a long time to develop, and also complicated and expensive testing gear. The same skills and equipment are probably needed to clear difficulties which might arise in production or in the field.

Units of this kind will probably be required for projects which may be undertaken in a number of different locations in the Laboratories' organization and at different times. It is therefore desirable that the functional responsibility for these units reside in a single permanent organization. The alternative would be to set up essentially small magnetics groups and it is unlikely that these could retain a continuity of personnel or interest over an extended period of time.

The compromise reached in the case of $\mathrm{TH}$, in other words, was not to be the general pattern for the future.

\section{Some Difficulties Not Observed in the TH Project}

Except for the difficulties associated with functional departments, the history of TH exhibits very few troubles which an organization patterned after a different ideal could have avoided. In particular, it fails to exhibit significantly the following troubles.

(1) Unnecessary effort, directed at (a) meeting requirements which are very difficult to fulfill and which could be modified to become easier without worsening the system, but are not; or (b) refinements of component design so as to exceed the requirements that suffice for satisfactory system performance ${ }^{33}$; or (c) unneeded parallel approaches to the component in question-approaches which do not serve to hedge against uncertainty since there exists a straightforward

${ }^{33}$ That is, refinements that either (1) permit attainment of system performance magnitudes superior to those chosen as system objectives but only at an effort which would not be considered worth making if the option arose as a conscious choice facing those responsible for the system's development; or (2) do not improve the performance magnitudes beyond those chosen as system objectives. 
predictable approach to designing the component so as to meet requirements.

(2) What appears ex post as a more or less chaotic scheduling of tasks-some tasks being finished long before the information they provide is needed, while the start of others has to await the completion of still other tasks that could have been started earlier.

(3) The performance of component work that turns out to be useless because the component in question poorly "matches" related components.

These troubles have been observed in other system development projects. ${ }^{34}$ While they require much further study it is fairly clear that their organizational pattern is different from the ideal pattern the TH project attempted to approximate. In important cases the pattern is something like the following. A system is planned at an early stage in considerable detail by a central authority. The authority schedules individual tasks and assigns them, together with an initial manpower allocation, to groups who, from lack of instructions to do so or because communication with the central authority is difficult, rarely question their assignments or suggest modifications. This leads to the difficulties described under 1 .

Moreover, the original detailed plan and schedule rest on predictions about the outcome of each group's effort. These predictions often turn out to be wrong, but the enforced insularity of each group makes it hard for the central authority to "catch" a bad prediction as to the difficulty of reaching an original goal until the group has expended considerable effort toward it. The group may have guessed at the magnitude of the predictive error some time earlier but does not bother the central authority until the situation is serious. At that point it may be possible to salvage the original system plan and schedule by a massive injection of manpower into the group in question, or it may be better to relax the goal of the group in question and make compensating adjustments in the rest of the system plan. In either case there is an additional cost or delay not expected when the original plan and schedule were approved, and the troubles 2 and 3 appear in the project's history.

Thus a project organization in which a central authority makes all the system decisions and parcels out tasks to groups who try to perform them in comparative isolation seems to make the project more susceptible than does the organizational pattern approximated by

${ }^{34}$ Notably certain military ones with which the writer has acquaintance. 
TH to the major difficulties observers have noted in system projectsoverstaffing (unnecessary effort), and gambles which expensively fail (heavy commitments to system plans and schedules based on unreliable initial knowledge). ${ }^{35}$

A project so organized might perhaps have advantages over one like $\mathrm{TH}$ in the luckiest possible case. If all predictions turned out exactly right, and a highly detailed plan and schedule had been based on them, then each group's initially assigned task would be precisely fulfilled. There would be no need for any communications apparatus to link the groups with the central authority, and no need for a systems analysis group to stand by, ready to work out modifications of the plan if they become necessary. But such lucky cases are rare indeed.

\section{Conclusion}

The essential features of the general strategy of development portrayed in the history may be briefly sketched as follows. The laboratory management decides on a ranking over the space of system performance magnitudes and system production and operating costs. It may be a very coarse ranking: there may be indifference within very large regions of the space. Corresponding to each region, nevertheless, is a maximum development effort (defined by a time path of expenditure, or perhaps a number of indifferent time paths), which the management is willing to expend in order to reach the region. There are generally several regions for which there is a relatively high confidence of attainment for not more (but not much less) than the associated maximum admissible effort. One of these regions is initially chosen as goal.

Within the chosen region, an initial set of performance magnitudes is then chosen as system objectives. The number of ways of attaining them may be enormous and only a few can be explored. First are considered several "families" of ways, each family defined by a

${ }^{35}$ A further phenomenon that some have observed in certain system projects is the growth of project staffs, from project to project within a laboratory, in accordance with some sort of Parkinson's Law, each increment in staff depending as much on previous size of staff as on the minimum effort actually required to complete the new project. If the type of project organization just described leads to the difficulties 1 to 3 , the project planners may decide that the only way to avoid these difficulties in the future is to be more liberal with manpower. This tends, however, to make the difficulties described under 1 more likely, since more manpower is now available for "unnecessary effort." Thus growth according to the Law may begin. 
set of system characteristics. Bit by bit, each family considered is narrowed, as the alternative component specifications consistent with each family are explored and knowledge of their difficulty is gained (using parallel design approaches to them where there is much uncertainty). Gradually a choice of family and of one way (one set of component specifications) within that family is made.

Until the final choice is made, flexibility is maintained. Commitments of personnel to specific tasks are made only for small periods, and it continues to be generally easy to drop one family or one way and to shift effort to exploring another more intensively. (Commitments are kept particularly small while awaiting sharp jumps in knowledge, such as completion of "breadboard" models may provide.) An exception are system-critical component specifications, which are "frozen" somewhat before the complete system is specified (before the final way is chosen), but only after a careful balancing of the risks and the gains. In the background remains, until the final choice, the possibility of changing the system objectives or even the region of indifference aimed for. When the system is finally specified, a schedule for the remaining development tasks is drawn up (to choose a good one, for a complex system, may be quite difficult).

A strategy of this sort-pursued against a background of continually accumulating experimental data and components, placed "on the shelf" for future systems-is necessary, we conjecture, if a given stream of expenditures on system development is to yield a stream of "successful" systems. A successful system project is one in which there are no major revisions at great cost - of which it cannot be said that much less effort would have been needed to attain the performance realized if only certain commitments had awaited knowledge gained latẹr in the system's development. An alternative strategyearly and massive commitment to one set of objectives and component specifications and one system schedule-will yield significantly more unsuccessful projects for the same expenditure stream..$^{36}$

These opposing strategies could be pursued, in principle, by a single man working on a project all his own. When other persons are

\footnotetext{
${ }^{36}$ One recent study concerns a planning tool to be used when this alternative strategy is followed. Development of a very complex system is considered as the completion of a huge array of highly specified tasks; the tool permits their efficient scheduling. Nowhere is the possibility considered that some of these tasks may turn out to be the "wrong" ones. See D. G. Malcolm, J. H. Rosebloom, C. E. Clark, and W. Fazar, "Application of a Technique for R and D Project Evaluation," Journal of the Operations Research Society of America, September-October, 1959.
} 
brought in, a choice of organizational pattern must be made. W further conjecture that the "ideal" organizational pattern describe above needs to be approximated in carrying out the first strateg described if a stream of successful projects is to be attained, wher the term unsuccessful is now enlarged to include specifically th difficulties (1) to (3) described above.

With this pair of informal conjectures, the experience of the $\mathrm{TI}$ project seems at least consistent. 